\title{
Enhancement of annihilation cross sections by electric interactions between the antineutron and the field of a large nucleus.
}

\author{
A. Bianconi , E. Lodi Rizzini, V.Mascagna , and L.Venturelli \\ Dipartimento di Ingegneria dell'Informazione, Università degli Studi di Brescia, I-25133 Brescia, Italy, \\ and Istituto Nazionale di Fisica Nucleare, Gruppo Collegato di Brescia \\ Email: andrea.bianconi@unibs.it \\ the date of receipt and acceptance should be inserted later
}

\begin{abstract}
Data relative to antineutron and antiproton annihilation on large nuclei in the range 75-200 $\mathrm{MeV} / \mathrm{c}$ present two unexpected features: (a) antineutron and antiproton cross sections have a similar size, (ii) the rise of the antineutron cross section at decreasing energy is much steeper than predictable for an inelastic process of purely strong nature at that energy. The observed behavior of $\bar{n}$-nucleus annihilations is similar to what would be expected for $\bar{p}$-nucleus annihilations, where Coulomb attraction focusses $\bar{p}$ trajectories towards the nucleus, enhancing the inelastic cross section by a factor $O(1 / p)$ with respect to $\bar{n}$ on the same target. This results in a $1 / p^{2}$ behavior at small energies. The presence of a similar enhancement in the antineutron case may only be justified by an interaction with a longer range than strong interactions. Excluding a Coulomb force because of the $\bar{n}$ neutrality, and taking into account that an intrinsic electric dipole is forbidden for the antineutron, the next choice is an electric dipole that is induced by the nuclear electric field. Recent theoretical works have shown that a non-negligible electric polarization may be induced in a neutron by QED vacuum polarization. Assuming this as a possibility, we have used a simple model to calculate the polarization strengths that are needed to fit the available data in terms of this effect. These are within the magnitude predicted by the vacuum polarization model. We have also discussed alternative scenarios that could induce an electric polarization of the antineutron as a consequence of the interplay between strong and e.m. interactions.
\end{abstract}

PACS. 12.20.-m Quantum electrodynamics - 24.80.+y Nuclear tests of fundamental interactions and symmetries - 25.43.+t Antiproton-induced reactions

\section{Introduction}

\subsection{Induced electric dipole in an antineutron}

Although the neutron (and equivalently the antineutron) is composed by constituents with nonzero electric charge, the existence of an intrinsic electric dipole moment for this particle is forbidden by general arguments. If the neutron had an electric dipole moment, $\mathrm{P}$ or $\mathrm{T}$ transformations would exchange the relative orientation of its magnetic and electric moments.

Since this rule is only valid in a $\mathrm{P}$ - or $\mathrm{CP}$-conserving context, many experiments have been performed to establish upper limits to this feature (see [1,2 for recent measurements, and $[3,4,5,6$ for reviews of the theoretical background and of the previous measurements). If an intrinsic dipole exists, it is very small.

In a CP-conserving context, a non-elementary particle may be polarized by an external electric field. No strict rule forbids this, because the relative orientation of the magnetic and electric moments in this case is not an intrinsic permanent property of the hadron. The most intuitive mechanism producing a polarization, that is a constituent charge displacement, requires mixing neutron internal states with opposite parity (see Sect. 4 for details). This may take place if these states are almost degenerate in mass, while the first negative parity state is several hundred $\mathrm{MeV}$ over the fundamental level.

In a recent theoretical work [7] it has been shown that QED corrections of order $\alpha^{2}$ (in the amplitude) permit an indirect interaction between the field of the neutron magnetic moment and an external electric field, mediated by vacuum polarization. As a consequence, the neutron behaves as if an electric dipole with strength proportional to the inducing field strength were present. This does not require displacements in the internal neutron charge distribution (this would be a lowest order QED effect). So it is a possible effect for an antineutron in its ground state. However, it requires an electric field whose strength is near the magnitude $10^{18} \mathrm{~V} / \mathrm{m}$ (Sauter's critical field [8]). The calculation of $[7$ is aimed at laser fields and motivated by 
the hope that lasers may approach this value in a near future. A later work [9] applies the same ideas to neutron elastic scattering by nuclei.

At the surface of a heavy nucleus the electric field has magnitude $10^{21} \mathrm{~V} / \mathrm{m}$ and a correspondingly large gradient. If an electric dipole may be induced in a neutron or an antineutron, short distance projectile-nucleus collisions are the place where this mechanism is strongest in our present possibilities. It is not clear to us at which extent the predictions by [7] and [9] may be adapted to the inelastic nuclear physics processes considered in the following. However, these give us a chance to consider the possibility that a response of some kind is induced in a slow antineutron by the electric field of a heavy nucleus. This would enhance the antineutron annihilation cross section at small energies, much as the Coulomb force does with antiprotons.

\subsection{Antineutron-nucleus and antiproton-nucleus annihilation cross section at small energies}

The data that are most relevant for the present work are the cross sections of antineutron annihilation on medium and large nuclei for projectile momentum $p<400 \mathrm{MeV} / \mathrm{c}$. These have been measured by several collaborations in the years 1980-2002 [10,11,12,13, 14 (see also [15] for a state-of-the-art review). The $\bar{n}$-nucleus annihilation data of [14] (see table 2 and fig.7 in that work) cover the range 76-380 MeV/c on several nuclear targets from carbon to lead, and are presently the available data reaching the smallest energy. For $p \geq 128 \mathrm{MeV} / \mathrm{c}$, the data from this experiment confirm very well previous data 13 and are almost perfectly fitted by an $a+b / p$ law, with a relevant contribution of the $b / p$ term. The smallest energy point at $76 \mathrm{MeV} / \mathrm{c}$ overrates by $15-20 \%$ this fit, for all the target nuclei, and an additional $1 / p^{2}$ term is needed to fit this last point.

In the case of the antiproton annihilations on light and heavy nuclei at low energies, many measurements have been performed in the region of momentum $p<$ $400 \mathrm{MeV} / \mathrm{c}$ (see Ref. [16, 17, 18, 19,20, 21, 22, 23, 24, 25, 26, 27, 28, 29]).

For $p>200 \mathrm{MeV} / \mathrm{c}$ antineutron and antiproton cross sections on the same target are almost equal (see fig.11 in [14). When momenta decrease below $200 \mathrm{MeV} / \mathrm{c}$ one expects $\bar{p}$-nucleus annihilation cross sections to grow much larger than antineutron ones, because of the Coulomb field of the nucleus pulling antiprotons towards the annihilation region from long distances $30,31,32,33,34$.

This dominance is well verified with data on light nuclear targets (in particular, with hydrogen targets, see fig. 1 in [35]), but has not been seen yet in annihilations on medium/large nuclear targets down to $76 \mathrm{MeV} / \mathrm{c}$. On the contrary, a compared analysis of $\bar{p}$-Sn [14] and $\bar{n}$-Sn [29] annihilation data below $100 \mathrm{MeV} / \mathrm{c}$, suggests that a low energy antineutron behaves in a more "electrostatic" way than an antiproton (see fig.6 in 35]).

Even ignoring the comparison between $\bar{p}$ and $\bar{n}$ data, it is difficult to explain the steep rise of the antineutron cross sections in the region $76-200 \mathrm{MeV} / \mathrm{c}$ in absence of medium/long range interactions, that is of interactions whose range is larger than the standard range of strong interactions. This point is better examined in Sect. 4.

In the following we assume that this interaction is of electromagnetic origin. Excluding Coulomb $\bar{n}$-nucleus interactions because of the overall neutrality of the antineutron, several possibilities are left, none of them obvious. The simplest one is an induced electric dipole. In Sect. 4 we will analyse its possible origin from several points of view. For the time being we just assume that it exists and use it to improve a basic fit of the data.

\section{Calculation method}

In the case of Coulomb interactions, the cross section enhancement with respect to a black sphere model may be calculated in a semiclassical way (see eq. 22 of [33]). With an electric dipole force the mathematics is more complex, so we will work numerically starting from a classic trajectory bending model. The procedure is the following.

1) The centre of the target nucleus is in the origin, and the projectile antineutron moves from the position $\left(0, y_{o},-\infty\right)$ with momentum $(0,0, p)$.

2) We assume that in absence of dipole effects the annihilation only involves antineutrons whose momentum is parallel to the $z$ axis until they reach the annihilation region. This region is assumed to be a sphere with radius $R_{\text {int }}=1.2 \cdot A^{1 / 3} \mathrm{fm}$. That is to say, only those antineutrons that "touch" the target nucleus may be involved in the annihilation process. This regards antineutrons with initial impact parameter $y_{o} \leq R_{\text {int }}$.

3) We assume that the interaction between these antineutrons and the nucleus is responsible for a cross section of the form $a+b / p$ exactly as given in eq.3 of [14], with the values of the parameters $a$ and $b$ from table 3 of that work $\left(a=66.5 \pm 3.0 \mathrm{mb}, b=(1.987 \pm 0.086) \cdot 10^{4} \mathrm{mb}\right.$ $\mathrm{MeV} / \mathrm{c})$. With all the used target nuclei this fit reproduces very well the measured cross section with the exception of the lowest point at $76 \mathrm{MeV} / \mathrm{c}$.

4) We introduce a long-distance force of the form $F(r)$ $=D(r) \partial E(r) / \partial r$, where $E(r)=Z e /\left(4 \pi \epsilon_{o} r^{2}\right)$ is the nuclear field and $D(r) \equiv \beta E(r)$ is the induced dipole. The polarizability $\beta$ is a constant parameter to be fitted.

5) We calculate the classical trajectories of the antineutrons in this field, out of the strong interaction region. since this field focusses projectile trajectories towards the target, the antineutrons reaching a target sphere of radius $1.2 A^{1 / 3} \mathrm{fm}$ have initial impact parameter $y_{o} \leq R_{d i p}$, with $R_{\text {dip }}>R_{\text {int }}$.

6) The fit $a+b / p$ (cross section without dipole) is multiplied by the correcting factor $R_{\text {dip }}^{2} / R_{\text {int }}^{2}$. This gives the "dipole-corrected cross section". We assume that this is the correct value of the cross section below $100 \mathrm{MeV} / \mathrm{c}$.

7) This procedure is repeated for several values of the polarization constant $\beta$, until we find a satisfactory reproduction of all the data of [14] for one nuclear target.

8) This calculation has been performed for the cases of $\mathrm{Cu}$ and $\mathrm{Sn}$ targets $(Z=29$ and 50 respectively, $A \approx 64$ 
Table 1. Fitted $L$ parameters. See text for details.

\begin{tabular}{|l|l|}
\hline \multicolumn{2}{|c|}{$L$ parameters } \\
\hline $\mathrm{Cu}$ & $3.8 \pm 0.7 \mathrm{fm}$ \\
$\mathrm{Sn}$ & $1.7 \pm 0.4 \mathrm{fm}$ \\
\hline
\end{tabular}

and 119 respectively after averaging over isotopes). Considering the very regular dependence of the data of 14 on the nuclear mass, we have no reasons to imagine that repeating the calculation for more targets would present surprises.

\section{Results}

The results are presented in figures $1(\bar{n}-\mathrm{Cu})$ and $2(\bar{n}-\mathrm{Sn})$. In each figure we report:

a) The data points by [14, and their $a+b / p$ fit that is very good on all the points for $p>100 \mathrm{MeV} / \mathrm{c}$.

b) Our dipole-corrected fit that allows fitting the 75 $\mathrm{MeV} / \mathrm{c}$ point without including an additional $1 / p^{2}$ term.

To give an understandable meaning to the fitted polarizability, we redefine the induced dipole moment as

$$
D(r)=\beta E(r) \equiv e Z L \frac{1 f m^{2}}{r^{2}}
$$

where $r$ is the distance of the antineutron to the nucleus center. If the nuclear electric field were emitted by a pointlike particle with charge $Z e$, at a distance $1 \mathrm{fm}$ from this particle the induced dipole would assume the value $e Z L$, physically readable as an electron and a positron separated by a distance $L Z$. The fitted values of $L$ are summarized in table 1 . The uncertainties are due to the error bars in the point at $76 \mathrm{MeV} / \mathrm{c}$ of each data set, visible in figs. 1 and 2 .

At the nuclear surface, where the dipole force is most relevant, the induced dipole is $e L Z / R_{\text {nucleus, } f m}^{2}$. These values imply $D=e .4 .8 \mathrm{fm}$ at the surface of a $\mathrm{Cu}$ nucleus, and $D=e \cdot 2.4 \mathrm{fm}$ at the surface of Sn. When these $D$ values are multiplied by $\partial E(r) / \partial r$ we find that the corresponding forces are roughly the same in the two cases.

These forces are medium range ones, that is longer than strong interactions but shorter than Coulomb ones. Within 1-2 fm of the nuclear surface they are almost as relevant as a Coulomb force: compared to the Coulomb force applied to an antiproton, at the Sn surface the antineutron dipole force is $9 / 10$, about $1 / 3$ at $2 \mathrm{fm}$ off the nuclear surface and $1 / 10$ at $6 \mathrm{fm}$. An analysis of the antineutron trajectories in our model shows that they are bended by dipole forces at distances of several fm, but serious deflections are confined to the last 2-3 fm of path before contact with the annihilation region.

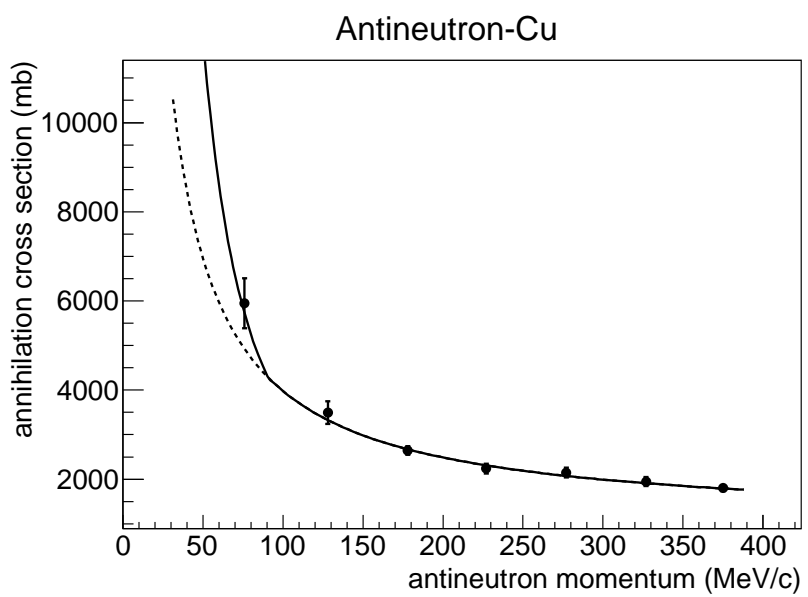

Fig. 1. Points with error bars: $\bar{n} \mathrm{Cu}$ annihilation data from 14. Dotted line: fit of the form $a+b / p$, with $b / a$ from table 3 of 14. Continuous line: dipole-corrected fit (see text). Remark: where the dotted line is not visible (for $p>100 \mathrm{MeV} / \mathrm{c}$ ), it is because of perfect overlap with the other curve.

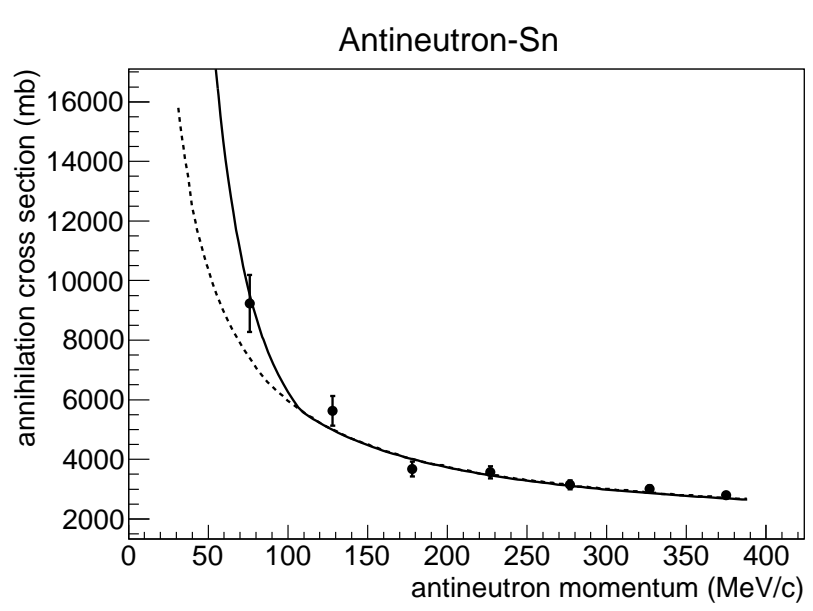

Fig. 2. Points with error bars: $\bar{n} \mathrm{Sn}$ annihilation data from 14. Dotted line: fit of the form $a+b / p$, with $b / a$ from table 3 of 14. Continuous line: dipole-corrected fit (see text). Remark: where the dotted line is not visible (for $p>100 \mathrm{MeV} / \mathrm{c}$ ), it is because of perfect overlap with the other curve.

\section{Discussion}

\subsection{Short and medium range interactions in the annihilation process}

The electric dipole force as fitted in our work behaves as a "medium range" one: if included in an optical model potential $U(r)+i W(r)$, it would imply a real negative (that is, attracting) term $U(r)$ with range about 2 fm larger than the imaginary (annihilating) term $W(r)$. Forgetting to interpret this interaction as an electric dipole force, our fits suggest the following consideration:

the $1 / p^{2}$-dependence of the data by 14 at small momenta, and the fact that the absolute value of the cross sections competes in magnitude with the corresponding antiproton cross sections, may be explained in terms of 
an elastic and attractive interaction that (i) in a range extending $2 \mathrm{fm}$ off the nuclear surface has the same size as a Coulomb interaction between charges $-e$ and $+Z e$, (ii) becomes rapidly negligible at larger distances.

Is a medium range interaction term really necessary to interpret the antineutron data? In our opinion it is, for the following reasons. Let us first assume a nonresonant behavior. The key parameters to qualitatively discuss this question are:

1) The diameter $d_{A, \text { strong }}$ of the strong interaction region, that is the nuclear diameter plus $1 \mathrm{fm}$ because of the range of the strong interactions. For Sn this means 12-13 $\mathrm{fm}$, and defines a maximum cross section $40 \mathrm{fm}^{2}$ for the region where the interactions directly responsible for the annihilation are effective.

2) $\hbar / p$, that at $100 \mathrm{MeV} / \mathrm{c}$ is $2 \mathrm{fm}$. This wavelength defines the borderline between a semiclassical regime and a fully quantum regime. Since the size of the region that is involved in the interaction cannot be smaller than the projectile wavelength because of the indetermination principle, if $\hbar / p>d_{A, \text { strong }}$ we are in a fully quantum regime. In this case a nonresonant inelastic cross section should follow a Bethe $1 / p$ law 30 . In the opposite regime $\hbar / p<$ $d_{A, \text { strong }}$ the cross section of the annihilation region cannot exceed much the geometrical cross section of the target nucleus. For $\mathrm{Sn}$ the borderline is $p \approx 30 \mathrm{MeV} / \mathrm{c}$, and for ${ }^{12} \mathrm{C} p \approx 70 \mathrm{MeV} / \mathrm{c}$. Since these borderlines are not sharp, it is reasonable to expect a small $1 / p$ contribution at $p \approx$ $100 \mathrm{MeV} / \mathrm{c}$, but not a steep rise.

3) The absorbing power of the elementary $\bar{n}$-nucleon interaction. In the considered energy range, the experimental $\bar{n}$-nucleon annihilation cross section is always $>$ $10 \mathrm{mb}$. The corresponding mean free path $\rho \sigma$ is $<1-2 \mathrm{fm}$. This means that the incoming antineutron flux is exponentially damped inside the target nucleus, with a damping range $<1-2 \mathrm{fm}$. With this premise a medium or large nucleus is a completely opaque sphere for an $\bar{n}$ flux at 200 $\mathrm{MeV} / \mathrm{c}$. The systematic increase of the $\bar{n}-p$ annihilation cross section below $200 \mathrm{MeV} / \mathrm{c}$ [36] cannot further increase this (already complete) opacity.

Any model that incorporates the above features will predict an almost constant $\bar{n}$-nucleus annihilation cross section for $\bar{n}$ momenta over $50 \mathrm{MeV} / \mathrm{c}$, of the form $\sigma \approx$ $\sigma_{\text {geometrical }}+b / p$ with a relatively small $b / p$ term. This is also what is is observed, but only for momenta over 200 $\mathrm{MeV} / \mathrm{c}$.

With a $\bar{p}$ projectile, the only difference is given by the Coulomb attraction. Since $Z e^{2} / r \approx 12 \mathrm{MeV}$ at the $\mathrm{Sn}$ surface, and $3 \mathrm{MeV}$ at the $\mathrm{C}$ surface, relevant effects of this interaction are not expected for projectile momenta $>>200 \mathrm{MeV} / \mathrm{c}$.

If we consider as an example ref. 35. (see fig.6 of that work) and ref. 31. (see fig.3 of that work), two independent model calculations confirm the previously described picture for antiproton and antineutron annihilations on medium-large nuclei in the region 50-400 MeV/c. Neither may explain the steep observed rise of the antineutron data in the momentum range $76-227 \mathrm{MeV} / \mathrm{c}$. Is it difficult to imagine a model predicting cross sections that qualitatively differ from the ones by [31] and 35, as far as this model implements nuclear interactions that are shortranged, present homogeneous features on the nuclear volume, and damp the projectile wavefunction within a range of 1-2 fm, on large nuclei at $p>50 \mathrm{MeV} / \mathrm{c}$. So one is obliged to introduce properties beyond this baseline modelling.

These cannot be related to features of one specific target. The data of [14] change in a regular way while passing from ${ }^{12} \mathrm{C}$ to very heavy nuclei. In all the considered momentum range including $76 \mathrm{MeV} / \mathrm{c}$, the measured cross sections may be fitted as $f(E) \cdot A^{x}$ with $x$ different from $2 / 3$ by less than $3 \%$ and $f(E)$ that does not depend on the target (see fig.9 in [14]). Although the neutron/proton ratio of the target nuclei ranges from 1:1 $(\mathrm{C})$ to $1.5: 1(\mathrm{~Pb})$, no dependence of the cross section on the neutron excess is seen. All this suggests that no effect is present that may be related to the features of a few specific nuclei, like a neutron halo. A resonance associated with the formation of a coherent $\bar{n}-A$ compound nucleus, or a set of overlapping resonances, would modify the cross section, but in a target-dependent way.

Resonance peaks could be present in the elementary $\bar{n} p$ and $\bar{n} n$ cross sections below $50 \mathrm{MeV} / \mathrm{c}$. The corresponding resonance in $\bar{n}$-nucleus would present a width $\sqrt{\Gamma_{r e s}^{2}+\Gamma_{F e r m i}^{2}}>200 \mathrm{MeV} / \mathrm{c}\left(\Gamma_{\text {res }}\right.$ is the resonance width, and $\Gamma_{\text {Fermi }} \approx 200 \mathrm{MeV} / \mathrm{c}$ is the effective width of the nuclear Fermi motion). This means that at $200 \mathrm{MeV} / \mathrm{c}$ the cross section should already present half of its resonance peak value (after subtracting from both the nonresonant $a+b / p$ background as fitted at larger momenta). So, the present data depend on energy more than a resonance can.

Summarizing the previous arguments, an explanation of the available data needs an interaction whose range is longer that the standard Yukawa strong interaction range, and whose strength is $\approx e^{2} / R_{\text {nucleus }}$ near the nuclear surface. This interaction must be systematically present in all the medium/large stable nuclear species, depending in a regular way on their gross features.

So, it is natural to think at electrostatic interactions. The point is whether physical mechanisms may be imagined, able to produce interactions of this class.

\subsection{Antineutron polarization via displacement of internal charges}

We first consider an electric dipole moment induced in the most obvious way: an opposite space displacement of the positive and negative antiquarks composing the antineutron, caused by the electric field surrounding the nucleus. If the internal position of an antiquark is given by the wavefunction $\Psi_{L}(\boldsymbol{r})$ with orbital angular momentum $L$, no left/right asymmetry is present in $\left|\Psi_{L}(\boldsymbol{r})\right|^{2}$. Such an asymmetry is present in $\left|a \Psi_{L 1}(\boldsymbol{r})+b \Psi_{L 2}\right|^{2}$ if $L 1$ and $L 2$ are angular momenta of opposite parity. Mixing the antineutron ground state with an opposite parity excited state requires an excitation energy of several hundred $\mathrm{MeV}$. This is impossible for a free or weakly interacting antineutron. 
The values of annihilation cross sections reported in the quoted references mean a mean path of magnitude less than $1 \mathrm{fm}$ before annihilation, for an antineutron inside the target nucleus. So, the antineutron is destroyed as soon as it touches the nucleus. On the other side, its constituent antiquarks keep travelling through the nucleus, binding themselves with nuclear quarks and forming mesons that further interact with the nuclear matter forming more fragments.

In other words, inside the nucleus the wavefunction describing the state of the 3 antiquarks loses its features of a bound state. This removes any constraint on the existence of an electric polarization for this set of 3 antiquarks. However, inside the nucleus the effects of an electric polarization cannot be disentangled from those of the strong interactions.

The question becomes whether a polarization mechanism that is allowed inside the nucleus may be effective at some distance off the nuclear surface, where strong interactions are not present. A regular interpolation must be present between the two wavefunctions describing the antiquarks out of the nucleus (where they form an antineutron, with all the described constraints forbidding a low-order QED polarization), and the antiquarks inside the nucleus (where no such constraints exist). The chances to find an electric polarization of an antineutron at some distance from the nuclear surface depend on the size of this interpolation region.

Summarizing this part, an induced electric dipole at lowest order QED in an antineutron near the surface of the target nucleus is not strictly forbidden, but a detailed annihilation model is required to quantify the strength of this effect.

\subsection{Antineutron polarization via vacuum polarization}

Let us consider the scheme proposed in 7]. At qualitative level this physical picture imagined for neutrons in quasistatic laser fields seems suitable for the case in study.

The intense value of the electric field at the Sn surface implies that the difference in electrostatic energy $U\left(r_{1}\right)$ - $U\left(r_{2}\right)$ is sufficient to create from the vacuum a $e^{+} e^{-}$ pair within a distance $r_{2}-r_{1} \approx 0.5 \mathrm{fm}$. This vacuum polarization effect is taken into account in ordinary charge renormalization in QED, that however assumes a pointlike and far test charge. Because of its proximity with the source of the nuclear field and of its finite diameter $>0.5$ $\mathrm{fm}$, an antineutron is able to test the local effects of this vacuum polarization.

In the scheme proposed by [7] the vacuum polarization pairs act as mediators of an indirect interaction between the field of the antineutron magnetic moment and the electric field of the nucleus. A necessary consequence is that the antineutron is able to test some features of the nuclear electric field. Saying this in other words, some electric multipoles are induced in the antineutron.

However unusual it may sound, the prediction that the magnetic field and the electric field produced by different particles may be coupled by vacuum polarization just belongs to the class of photon-photon interactions, like $\gamma \gamma$ $\rightarrow X$ experiments at $e^{+} e^{-}$colliders, for which decades of experimental results exist since [37,38,39. The important difference is that in the collider case the local fields are so intense to permit vacuum fluctuations into real $\bar{q} q$ pairs with involvement of the strong interactions and large enhancement of the cross sections. So a sensitivity of the antineutron to the nuclear electric field cannot be excluded, the real problem being its strength.

The induced dipole value given by eq.29 of [7] depends on a length parameter " $a$ " (the dipole is $\propto 1 / a^{3}$ ). In 7 $a$ parameterized a charge current distribution in the neutron, and values of $a \leq 1 \mathrm{fm}$ were suggested. In a later work 9] a was reinterpreted as a parameter incorporating the effects of higher order corrections and values of $a$ over $7 \mathrm{fm}$ were suggested. Given the value of the electric field of the Sn nucleus, we obtain the dipole value fitted by us for $a \approx 2 \mathrm{fm}$.

\subsection{Nonlinearity}

As fitted by us, the difference between the polarizabilities in the $\mathrm{Cu}$ and $\mathrm{Sn}$ cases means that we are out of a linear regime, where the induced dipole would be proportional to the inducing field. In our case a stronger field induces a dipole that is weaker than a linear law expectation. We may speak of "strong-field saturation".

If the induced dipole is associated to a stretching of the projectile internal structure this saturation is natural, since QCD interactions between the hadron constituents are well known to be small at distances $<<1 \mathrm{fm}$ but rapidly increasing at distances $\sim 1 \mathrm{fm}$. These would oppose to an antineutron dipole with strength $e \delta$ for $\delta>1$ $\mathrm{fm}$, saturating the polarization at large $\delta$. More in general, it is unavoidable that a force acting on the antineutron as a whole brings some stress on the internal structure of this hadron. When this stress implies deformations of size $>1$ fm, QCD interactions will introduce nonlinearity.

Within QED, the effects as calculated by [7] correspond to a given power of $\alpha$ (see eq. 2 in that work). Extending to higher orders would introduce some degree of negative feedback at increasing fields because of Lenz's law. This would naturally show as a smaller polarizability in presence of a larger inducing field. This nonlinearity must be expected for $E>>10^{18} \mathrm{~V} / \mathrm{m}$ (the Sauter's critical value) because then the $e^{+} e^{-}$pairs proliferate instead of being a small perturbation, and their reciprocal screening cannot be neglected.

In conclusion, it is reasonable to expect nonlinearity when the dipole value corresponds to $e L$ with $L>1 \mathrm{fm}$ because of QCD confinement. It is also reasonable to expect nonlinearity when the inducing field is $>>10^{18} \mathrm{~V} / \mathrm{m}$ because of QED higher order terms and Lenz's law. And in both cases this nonlinearity is expected to lead towards saturation. 


\section{Conclusions and perspectives}

The main conclusion of this note is that a medium range force, in our assumption of electromagnetic origin, allows for a physically based justification of the $1 / p^{2}$ term in the fit of the antineutron annihilation cross section on medium and large nuclei at 76-375 MeV/c. Exploiting recent analysis claiming the existence of an induced electric dipole moment in the neutron, we have assumed that an analogous moment is induced in an antineutron by the electric field of a nearby nucleus. The attracting force deriving from this leads to a steep energy dependence of the annihilation cross section for projectile momenta below $100 \mathrm{MeV} / \mathrm{c}$. We have presented arguments to demonstrate that a medium range interaction is necessary for justifying the steep rise of the cross section in the region $50-200 \mathrm{MeV} / \mathrm{c}$.

In perspective, our work suggests the need for 1-2 more antineutron data points that permit to estimate the exact slope of the $\bar{n}$-nucleus cross section in the region 50-100 $\mathrm{MeV} / \mathrm{c}$, and understand where exactly an $a+b / p$ law must be abandoned. This would put more selective constraints on the form (strength, range and asymptotic behavior) of the involved medium-range interaction term.

An analysis of elastic data, as suggested in 9, could also shed light on these points. In the energy region considered in this work, data on the differential distribution of elastically scattered antinucleons are still absent.

A relevant open point with these data, presently admitting neither an intuitive justification nor one from a complex model, is the relative size of the $\bar{n}$-nucleus and $\bar{p}$-nucleus annihilation cross sections for $p<100 \mathrm{MeV} / \mathrm{c}$. Since in this moment the antineutron data in this region are more systematic and precise than the antiproton ones, an as detailed as possible exploration of the antiproton annihilation on nuclei below $100 \mathrm{MeV} / \mathrm{c}$ is required. In particular what is missing is a detailed interpolation between the behavior of the antiproton annihilation on light nuclei like hydrogen or helium (where a very steep Coulomb rise is visible, and antiproton cross sections overcome antineutron ones by a factor as large as three) and on medium and large nuclei where we find cross sections that seem far smaller than expected. Ideally, a precision that allows to distinguish constant, $1 / p$ and $1 / p^{2}$ components of the cross section $p$-dependence is required.

\section{References}

1. C.A.Baker et al, Phys.Rev.Lett.97, 131801 (2006).

2. V.V.Fedorov et al, Phys.Lett.B 694, 22-25 (2010)

3. S.Dar, arXiv:hep-ph/0008248

4. M.Pospelov and A.Ritz, Annals Phys.318:119-169,2005

5. J.C.Peng, Mod.Phys.Lett. A23, 1397-1408 (2008).

6. S.K. Lamoreaux and R. Golub, J. Phys. G 36104002 (2009).

7. C.A.Dominguez, H.Falomir, M.Ipinza, S.Kohler, M.Loewe, and J.C.Rojas, Phys.Rev.D 80, 033008 (2009).

8. F. Sauter, Z. Phys. 69, 742-764 (1931).

9. O.Zimmer, C.A.Dominguez, H.Falomir, and M.Loewe, Phys.Rev.D 85, 013004 (2012).
10. B. Gunderson et al., Phys. Rev. D 23 (1981) 587.

11. M. Agnello et al., Europhys. Lett. 7 (1988) 13.

12. C. Barbina et al., Nucl. Phys. A 612 (1997) 346.

13. V.G. Ableev et al., Nuovo Cimento 107 A (1994) 943.

14. M. Astrua et al., Nucl. Phys. A 697 (2002) 209224

15. T.Bressani and A.Filippi, Phys. Rep. 383213297 (203).

16. R. Bizzarri et al., Nuovo Cim. A22 (1974) 225

17. T.E. Kalogeropoulos and G.S. Tzanakos, Phys. Rev. 22D (1980) 2585

18. F. Balestra et al., Phys. Lett. B 149 (1984) 69

19. F. Balestra et al., Phys. Lett. B 165 (1985) 265

20. F. Balestra et al., Nucl. Phys. A 452 (1986) 573

21. F. Balestra et al., Phys. Lett. B 230 (1989) 36

22. W. Brückner et al., Z. Phys. A 335 (1990) 217

23. A. Bertin et al. (OBELIX Collaboration), Phys. Lett. B 369 (1996) 77

24. A. Benedettini et al. (OBELIX Collaboration), Nucl. Phys. B Proc. Suppl. 56A (1997) 58

25. A. Zenoni et al. (OBELIX Collaboration), Phys. Lett. B 461 (1999) 405

26. A. Zenoni et al. (OBELIX Collaboration), Phys. Lett. B 461 (1999) 413

27. A. Bianconi et al., Phys. Lett. B 481 (2000) 194

28. A. Bianconi et al., Phys. Lett. B 492 (2000) 254

29. A. Bianconi et al., Phys. Lett. B 704 (2011) 461.

30. L. D. Landau, E. M. Lifshitz, "Course of Theoretical Physics - Quantum Mechanics (Non-Relativistic Theory)" Butterworth-Heinemann Vol 3

31. A.Bianconi, G.Bonomi, E.Lodi Rizzini, L.Venturelli and A.Zenoni, Phys.Rev. C62 014611 (2000).

32. A.Bianconi, G.Bonomi, M.P.Bussa, E.Lodi Rizzini, L.Venturelli and A.Zenoni, Phys.Lett. B 483 353-359 (2000).

33. C.J.Batty, E.Friedman, and A.Gal, Nucl.Phys. A 689 721740 (2001).

34. J.Carbonell and K.V.Protasov, Hyp. Int. 76327 (1993).

35. E.Friedman, Nucl. Phys. A 925 (2014) 141149

36. F.Iazzi, et al., Phys. Lett. B 475 (2000) 378.

37. C.Bacci et al, Proc. First EPS conf. on meson resonances and related electromagnetic phenaoena, Bologna, 1971. C.Bacci et al, Lett. Nuovo Cim. 3, 709 (1972).

38. C.H.Berger et al, Phys.Lett. B, 120 (1979).

39. S.E.Baru et al, Z.Phys.C 53219 (1992). 